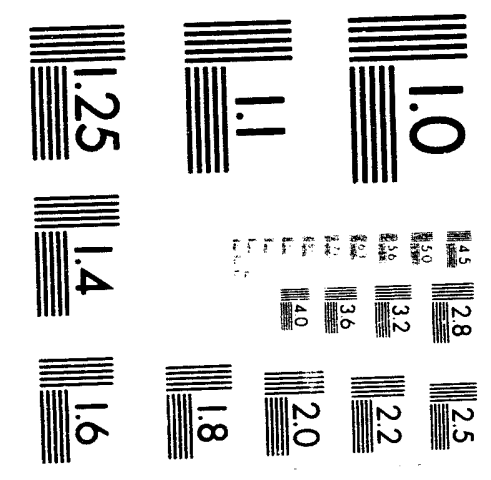



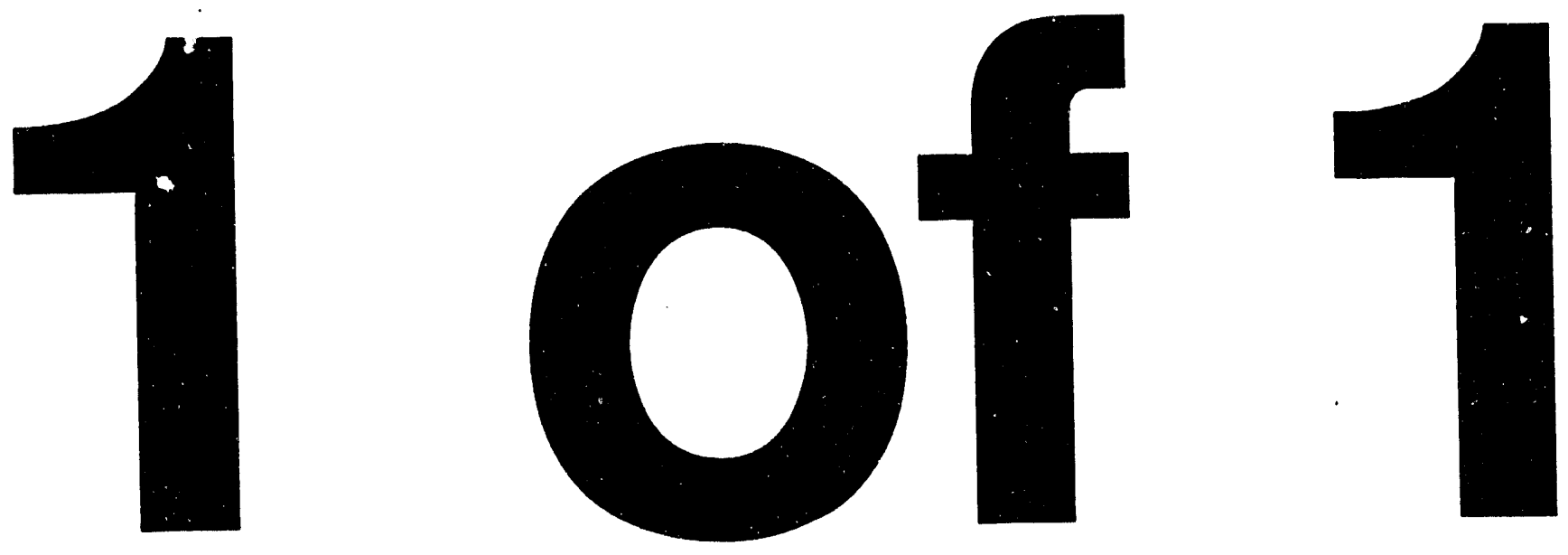


\title{
PHOTONIC BAND GAP MATERIALS
}

\author{
C. M. SOUKOULIS
}

Ames Laboratory and Department of Physics

and Astronomy, Iowa State University, Ames, IA 50011 USA.

ABSTRACT. An overview of the theoretical and experimental efforts in obtaining a photonic band gap, a frequency band in three-dimensional dielectric structures in which electromagnetic waves are forbidden, is presentea.

Electron waves traveling in the periodic potential of a crystal are arranged into energy bands separated by gaps in which propagating states are prohibited[1]. It is interesting to see if analogous band gaps exist when electromagnetic (EM) waves propagate in a periodic dielectric structure (e.g., a periodic lattice of dielectric spheres of dielectric constant $\epsilon_{a}$ embedded in a uniform dielectric background $\epsilon_{b}$ ). If such a band gap or frequency gap exists, EM waves with frequencies inside the gap cannot propagate in any direction inside the material. These frequency gaps are referred to as "photonic band gaps."

Photonic band gaps can have a profound impact on many arcas in pure and applied physics[2,3]. Due to the absence of optical modes in the gap, spontaneous emission is suppressed for photons with frequencies in the forbidden region[4,5]. It has been suggested that, by tuning the photonic band gap to overlap with the electronic band edge, the electron-hole recombination process can be cortrolled in a photonic band gap material, leading to enhanced efficiency and reduced noise in the operation of semiconductor lasers and other solid state devices $[3,5]$. The suppression of spontaneous emission can also be used to prolong the lifetime of selected chemical species in catalytic processes[6]. Photonic band gap materials can also find applications in frequency-selective mirrors, band-pass filters, and resonators. Besides technical applications in various areas, scientists are interested in the possibility of observing the localization of EM waves by the introduction of defects and disorder in a photonic band gap material[7-9]. This will be an ideal realization of the phenomenon of localization uncomplicated by many-body effects present in the case of electron localization. Another interesting effect is that, zero-point fluctuations, which are present even in vacuum, are absent for frequencies inside a photonic gap. Electromagnetic interaction governs many properties of atoms, molecules, and solids. The absence of EM modes and zero point fluctuations inside the photonic gap can lead to unusual physical phenomena[7-12]. For example, atoms or molecules embedded in such a

\section{DISCLAIMER}

OISTRIBUTION OF THIS DOCUMENT IS UNLIMITED

This report was prepared as an account of work sponsored by an agency of the United States Government. Neither the United States Government nor any agency thereof, nor any of their employees, makes any warranty, express or implied, or assumes any legal liability or responsibility for the accuracy, completeness, or usefulness of any information, apparatus, product, or process disclosed, or represents that its use would not infringe privately owned rights. Reference herein to any specific commercial product, process, or service by trade name, trademark, manufacturer, or otherwise does not necessarily constitute or imply its endorsement, recommendation, or favoring by the United States Government or any agency thereof. The views and opinions of authors expressed herein do not necessarily state or reflect those of the United States Government or any agency thereof.

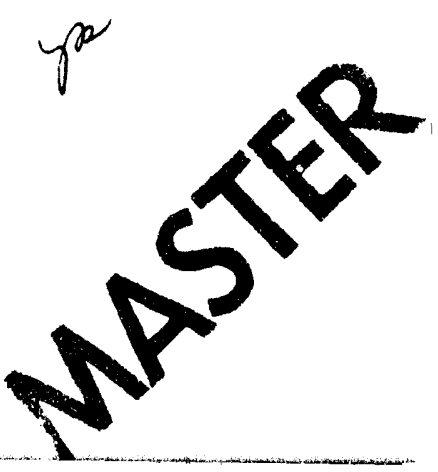


material can be locked in excited states if the photons emitted to release the excess energy have frequency within the forbidden gap. All the aforementioned ideas[3] about new physics and new technology hinge upon the assumption of the existence of material with photonic gaps.

To search for the appropriate structures, scientists at Bellcore employed a "cutand-try" approach in which various periodic dielectric structures were fabricated in the microwave regime and the dispersion of EM waves were measured to see if a frequency gap existed[13]. The process was time consuming and not very successful. After attempting dozens of structures over a period of two years, Yablonovitch and Gmitter identified[13] only one structure with a photonic band gap. This structure consists of a periodic array of overlapping spherical holes inside a dielectric block. The centers of the holes are arranged in a face-centered-cubic (fcc) lattice and the holes occupy $86 \%$ of the volume of the block.

Stimulated by the experimental work, theorists became interested in the solution of the photonic band problem and in the search for structures with photonic band gaps. Early work in this area employed the "scalar wave approximation" which assumed the two polarizations of the EM waves can be treated separately, thus decoupling the problem into the solution of two scalar wave equations. When we first became involved with the photon band problem, calculations had already been completed for the experimental structure in the scalar wave approximation[14,15]. The results showed the existence of a gap but the position and size of the gap were not in quantitative agreement with the experiment, indicating the nced for a full vector wave treatment. It turned out from subsequent calculations that the errors made in neglecting the vector nature of the EM wave were more serious than initially anticipated, and the scalar wave calculations actually gave qualitatively wrong results.

The vector wave solution of Maxwell's equations for a periodic dielectric system was carried out independently by several groups shortly after the appearance of the scalar wave results[16-18]. All of the methods employ a plane wave expansion of the electromagnetic fields and use Bloch's theorem to reduce the problem to the solution of a set of linear equations.

When the photon band structure for the experimental fcc structure[13] of $86 \%$ air spheres in a dielectric matrix, was calculated[18], it showed that the experimental fcc structure does not have a complete photonic band gap for the lowest-lying bands. A very large depletion of DOS is found, called a "pseudo-gap." Actually, this result was also obtained earlier by two other groups[16,17], although at that time we were not aware of their results. At this point, the existence of photonic gap materials was seriously doubted[19]. However, since we found that the plane wave expansion method[16-18] can solve the photon band problem efficiently and much faster than the experimental "cut-and-try" method, we used it to investigate whether other structures could succeed where the fcc air sphere structure failed. 
Ho, Chan, and Scukoulis were the first to give a prescription for a periodic dielectric structure[18] that possesses a full photonic band gap rather than a pseudogap. This proposed structure is a periodic arrangement of dielectric spheres in a diamondlike structure. A systematic examination[18] of the photonic band structures for dielectric spheres and air spheres on a diamond lattice, as a function of the refractive index contrasts and filling ratios, was made. It was found that photonic band gaps exist over a wide region of filling ratios fcr both dielectric spheres and air spheres for refractive-index contracts as low as 2 . However, this diamond dielectric structure is not easy to fabricate, especially in the micron and submicron length scales for infrared or optical devices. However, after we communicated our findings about the diamond structure, Yablonovitch very quickly devised[20] an ingenious way of constructing a diamond lattice. He noted that the diamond lattice is a very open structure characterized by open channels along the $[110]$ directions. Thus, by drilling cylindrical holes through a dielectric block, a structure with the symmetry of the diamond structure can be created. Since there are 6 sets of equivalent [110] directions in the lattice, there are 6 sets of holes drilled. If the crystal is oriented such that the [111] surface is exposed, then three sets of these holes will be slanted at angles of $35.26^{\circ}$ with respect to the normal [111] direction. The remaining three sets of holes have their axes parallel to the [111] surface and are harder to make on a thin film oriented in the [111] direction. Thus, in the end, the experimentalists decided to abandon the second three sets of holes and construct a structure with only the first three sets of holes (see Fig. 15, in the article by Yablonovitch in Ref. 3) which became the first experimental structure that demonstrates the existence of a photonic band gap, in agreement with the predictions[21] of the theoretical calculations. This is a successful example where the theory was used to design dielectric structures with desired properties.

We repeated our calculations for several variations on the diamond lattice[21]. One calculation uses the diamond lattice generated by 6 sets of air cylinders or dielectric cylinders in the six [110] directions. The other calculation uses a diamond rod lattice in which, instead of putting spheres at the lattice sites, we joined them together by nearest-neighbor rods. We also tested the effects on the photon band gap when 3 sats of cylinders are omitted in the 6-cylinder diamond structure. All of these structures exhibit photonic band gaps, with the best performance coming from a diamond rod lattice, which achieves a maximum gap of $30 \%$ for a refractive index contrast of 3.6 .

Very narrow photonic band gaps have also been found[22] in a simple cubic geometry. For 2D systems[23-25], theoretical studies have shown[23,24] that a triangular lattice of air columns in a dielectric background is the best overall 2D structure, which gives the largest photonic gap with the smallest refractive index contrast. In addition, it was demonstrated[26-29] that lattice imperfections in a 2D and/or 3D periodic arrays of a dielectric material can give rise to fully localized EM wave functions. Experimental inves igations of the photonic band gaps in either 2D or 3D have been mostly done $[20,26,28,29]$ at microwave frequencies because of the difficulty in 
fabricating ordered dielectric structures at optical length scales. In fact, the main challenge in the photonic band gap field is the discovery of a 3D dielectric structure that exhibits a photonic gap but, in addition, can be built by microfabrication techniques on the scale of optical wavelengths. The Iowa State University group of Ho, Chan, and Soukoulis recently introduced a new 3D layer structure that has the potential in solving the only outstanding problem in the field. The creation of a photonic gap in a microstructure amenable to conventional microfabrication.

All of the theoretical results discussed above were obtained with the plane-wave expansion technique, which is now very well developed. However, most of the theoretical techniques concentrate on the calculation of the dispersion of the photon bands in the infinite periodic structure, while experimental investigations focus mainly on the transmission of EM waves through a finite slab of the photonic band gap patterned in the required periodic structure. Even with the knowledge of the photon band structure, it is still a non-trivial task to obtain the transmission coefficient for comparison with experiment. Another important quantity for the photonic band gap experiments and devices is the attenuation length for incident EM waves inside the photonic band gap. Another topis of interest is the behavior of impurity modes associated with the introduction of defects into the photonic band gap structure. While this problem can be tackled within a plane wave approach using the supercell method[26,27] in which a simple defect is placed within each supercell of an artificially periodic system, the calculations require a lot of computer time and memory. Recently, Pendry and MacKinnon[30] introduced a complimentary technique for studying photonic band gap structures. Their method has the advantage that the transmission coefficients and attenuation coefficients for the incident EM waves for various frequencies can be obtained directly from the calculations. We have also calculated the transmission coefficient for 2D and 3D structures with excellent agreement with experiment.

We want to stress that this technique can also be applied to cases where the planewave method fails or becomes too time consuming. For example, when the dielectric constant is frequency dependent, or has a non-zero imaginary part, and when defects are present in an otherwise periodic system, this technique works well.

In summary, we have revicwed the theoretical and experimental efforts in obtaining $2 \mathrm{D}$ and $3 \mathrm{D}$ dielectric structures that possess a full photonic band gap. The plane-wave method results of $\mathrm{Ho}, \mathrm{Chan}$, and Soukoulis suggested the first structure to exhibit a true photonic band gap, and the Yablonovitch "3-cylinder" structure of diamond symmetry was the first experimental structure with a photonic band gap. We have demonstrated that a systematic search for the structures that possess optimal photonic gaps can be conducted via theoretical calculations. Practical three-dimensional periodic arrangements of dielectric structures were proposed. These dielectric structures possess a full photonic gap, with refractive-index contrasts as low as 1.9 , and are much easier to fabricate than dielectric spheres arranged in a diamond lattice. While there is no simple explanation as to the exact conditions where photonic band 
gaps appear, our findings suggest that the existence or non existence of gaps is related to the connectivity of the high dielectric material and the symmetry of the resulting structure. We are excited about the future applications of photonic band gaps and the prospects of using our calculational techniques to design and help the fabrication of these photonic band gap materials.

\section{ACKNOWLEDGMENTS}

This work was done in collaboration K .M. Ho and C. T. Chan. Ames Laboratory is operated by the U.S. Department of Energy by lowa State University under Contract No. W-7405-Eng-82. This work was supported by the Director for Energy Research, Office of Basic Energy Sciences and Advanced Energy Projects and by NATO Grant No. RG769/87.

\section{REFERENCES}

[1]. See e.g., C. Kittel, Introduction of Solid State Physics, (5th Edition, Wiley, 1976) Ch. 7.

[2]. See the proceedings of the NATO ARW, Photonic Band Gaps and Localization, ed. by C. M. Soukoulis, (Plenum, N.Y., 1993).

[3]. For a recent review see the special issue of J. Opt. Soc. Am. B 10, 280-408 (1993) on the Development of Applications of Materials Exhibiting Photonic Band Gaps.

[4]. E. M. Purcell, Phys. Rev. 69, 681 (1946).

[5]. E. Yablonovitch, Phys. Rev. Lett. 58, 2059 (1987).

[6]. N. Lawandy, in Photonic Band Gaps and Localization, ed. by C. M. Soukoulis (Plenum Publ., N.Y., 1993), p. 355

[7]. S. John, Phys. Rev. Lett. 58, 2486 (1987); S. John, Comments, Cond. Matt. Phys. 14, 193 (1988); S. John, Physics Today 32, 33 (1991).

[8]. Scattering and Localization of Classical Waves in Random Media, ed. by P. Sheng (World Scientific, Singapore, 1990).

[9]. J. M. Drake and A. Z. Genack, Phys. Rev. Lett. 63, 259 (1989).

[10]. C. A. Condat and T. R. Kirkpatrick, Phys. Rev. B 36, 6783 (1987).

[11]. J. Martorell and N.'M. Lawandy, Phys. Rev. Lett. 65, 1877 (1990).

[12]. G. Kurizki and A. Z. Genack, Phys. Rev. Lett. 66, 1850 (1991).

[13]. E. Yablonovitch and T. J. Gmitter, Phys. Rev. Lett. 63, 1950 (1989).

[14]. S. Satpathy, Z. Zhang, and M. R. Salehpour, Phys. Rev. Lett. 64, 1239 (1990).

[15]. K. M. Leung and Y. F. Liu, Phys. Rev. B 41, 10188 (1990). 
[16]. K. M. Leung and Y. F. Liu, Phys. Rev. Lett. 65, 2646 (1990).

[17]. Z. Zhang and S. Satpathy, Phys. Rev. Lett. 65, 2650 (1990).

[18]. K. M. Ho, C. T. Chan, and C. M. Soukoulis, Phys. Rev. Lett. 65, 3152 (1990).

[19]. J. Maddox, Nature 348, 481 (1990).

[20]. E. Yablonovitch, T. J. Gmitter, and K. M. Leung, Phys. Rev. Lett. 67, 2295 (1991); E. Yablonovitch and K. M. Leung, Nature 351, 278 (1991).

[21]. C. T. Chan, K. M. Ho, and C. M. Soukoulis, Europhys. Lett. 16, 563 (1991).

[22]. H. S. Sözuer and J. W. Haus, J. Opt. Soc. Am. B 10, 296 (1993) and references therein.

[23]. P. R. Villeneuve and M. Piche, Phys. Rev. B 46, 4964 (1992); ibid 46, 4973 (1992).

[24]. R. D. Meade, K. D. Brommer, A. M. Rappe, and J. D. Joannopoulos, Appl. Phys. Lett. 61, 495 (1992).

[25]. M. Plihal, A. Shambrook, A. A. Maradudin, and P. Sheng, Opt. Commun, 80, 199 (1991); M. Plihal and A. A. Maradudin, Phys. Rev. B 44, 8565 (1991).

[26]. E. Yablonovitch, T. J. Gmitter, R. D. Meade, A. M. Rappe, K. D. Brommer, and J. D. Joannopoulos, Phys. Rev. Lett. 67, 3380 (1991).

[27]. R. D. Meade, K. D. Brommer, A. M. Rappe, and J. D. Joannopoulos, Phys. Rev. B 44, 13772 (1991).

[28]. S. L. McCall, P. M. Platzman, R. Dalichaouch, D. Smith and S. Schultz, Phys. Rev. Lett. 67, 2017 (1991); S. Schultz and D. R. Smith, to be published.

[28]. W. Robertson, G. Arjavalingan, R. D. Meade, K. D. Brommer, A. M. Rappe and J. D. Joannopoulos, Phys. Rev. Lett. 68, 2023 (1992).

[30]. J. B. Pendry and A. MacKinnon, Phys. Rev. Lett. 69, 2772 (1992). 

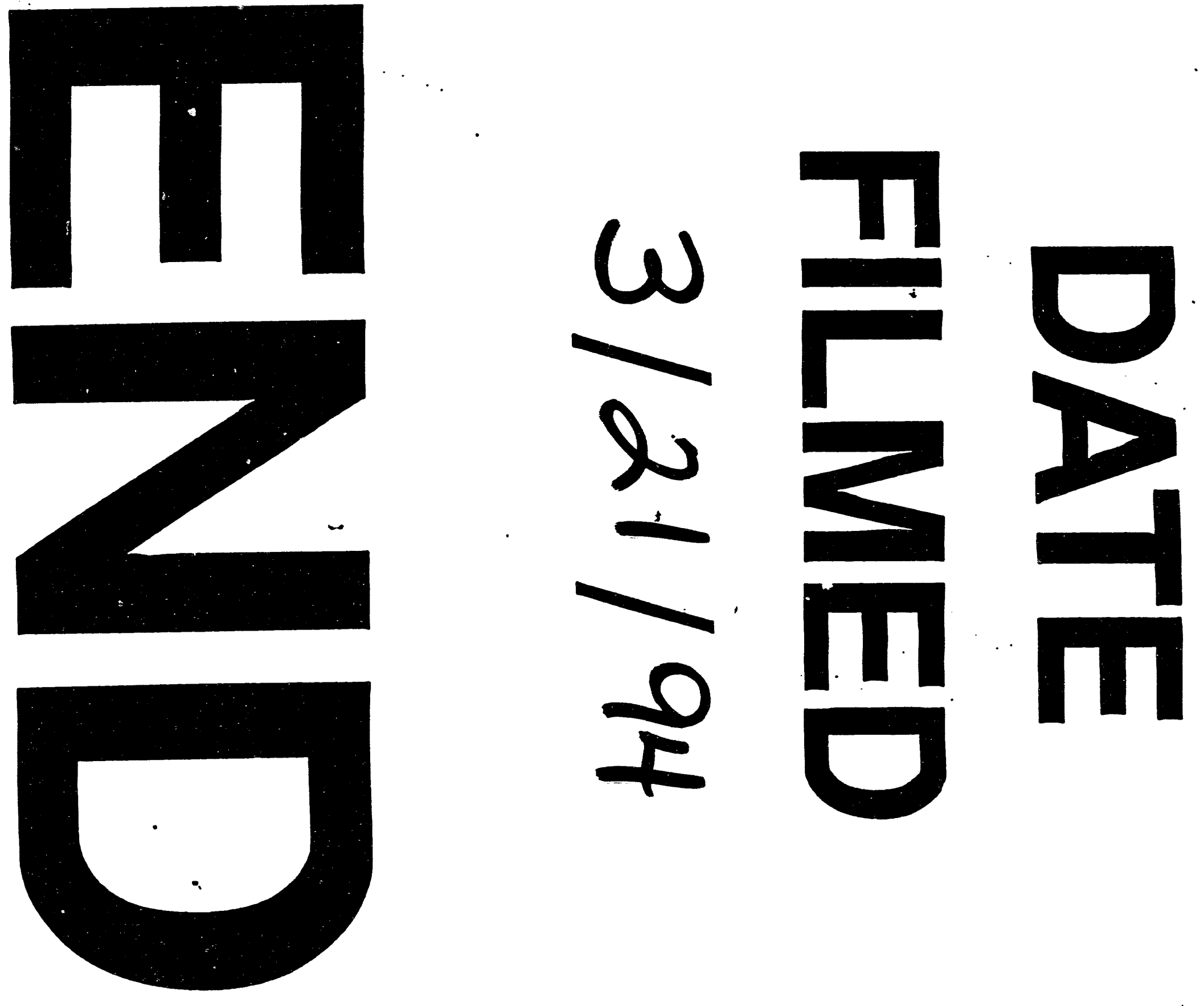
